\title{
REVISÃO SISTEMÁTICA: UMA REVISÃO NARRATIVA
}

\author{
SYSTEMATIC REVIEW: A NARRATIVE REVIEW
}

\author{
Alexander Magno Cordeiro'; Glória Maria de Oliveira ${ }^{2}$; \\ Juan Miguel Rentería - TCBC-RJ33 ${ }^{3}$ Carlos Alberto Guimarães - TCBC- RJ ${ }^{4}$; \\ Grupo de Estudo de Revisão Sistemática do Rio de Janeiro (GERS-Rio) ${ }^{5}$
}

\begin{abstract}
RESUMO: Objetivo: realizar uma revisão narrativa sobre revisão sistemática. Fonte dos dados: foi realizada busca no Cochrane Methodology Register, na Medline, na LILACS, no Google Scholar e na Cochrane Library, no período de 2000 a janeiro de 2007. Foram utilizados a busca manual das listas de referências e os contatos pessoais. Seleção dos estudos: a estratégia de busca empregou, na Medline, as seguintes combinações dos termos MeSH: "Meta-Analysis" [Publication Type] AND "Evidence-Based Medicine"[MeSH] Limits: Publication Date from 2000 to 2007, Humans, Systematic Reviews. Na LILACS: (metanalise) or "metanalise" [Descritor de assunto] and [ medicina baseada em evidências] or "medicina baseada em evidências" [Descritor de assunto]. No Cochrane Methodology Register e no Google Scholar: "revisão sistemática e metanálise" e "medicina baseada em evidências". Após uma revisão independente por dois revisores, dez artigos que se referiam ao objetivo proposto foram selecionados. Síntese dos dados: os temas mencionados nos estudos foram agrupados em duas categorias: aqueles que se reportavam à história da revisão sistemática e aqueles que definiam Medicina Baseada em Evidência, revisão sistemática e metanálise. Conclusão: os autores concluem com a necessidade de mais discussões sobre revisão sistemática entre os cirurgiões (Rev. Col. Bras. Cir. 2007; 34(6): 428-431).
\end{abstract}

Descritores: Comunicação. Revisão sistemática. Metanálise. Literatura de revisão. Revisão narrativa. Medicina Baseada em Evidências.

\section{INTRODUÇÃO}

Nas últimas décadas, o grande volume das informações científicas geradas na área da Saúde aponta para a necessidade de sínteses que facilitam o acesso às mesmas, possibilitando conclusões baseadas na combinação dos resultados oriundos de múltiplas fontes.

As práticas baseadas em evidência, apesar de não estarem isentas de críticas, podem contribuir para a consistência da fundamentação de uma decisão clínica. A Medicina Baseada em Evidências, assim com os meios de praticá-la, tem sido foco de atenção, em que se destacam as revisões sistemáticas com ou sem metanálise.

Neste trabalho, os autores apresentam uma revisão narrativa dos conceitos que definem Medicina Baseada em Evidência, revisão sistemática com e sem metanálise e revisão narrativa.

\section{MEDICINA BASEADA EM EVIDÊNCIA}

Medicina Baseada em Evidência (MBE) pode ser definida como o uso consciencioso, explícito e judicioso das melhores evidências na tomada de decisões relativas à prática com pacientes individuais, unindo evidências produzidas em pesquisa com experiência clínica ${ }^{1}$.
A MBE não exclui a experiência e o conhecimento clínico individual, no entanto preconiza que esses devem estar associados às evidências objetivas ${ }^{1}$. As primeiras noções sobre MBE foram desenvolvidas no Canadá, porém esse tipo de prática tem tido maior impacto na Grã- Bretanha, tendo em vista sua adoção pelo sistema público de saúde.

A MBE utiliza as ferramentas da Epidemiologia Clínica, da Estatística, da Metodologia Científica e da Informática, para trabalhar a pesquisa, com o objetivo de oferecer a melhor informação disponível para a tomada de decisão.

Na prática clínica, quatro situações básicas se repetem: diagnóstico, tratamento, prognóstico e prevenção. Cada situação compreende cinco componentes específicos: acesso à informação, avaliação crítica da literatura, principais desenhos de pesquisa clínica, métodos estatísticos e planejamento da pesquisa clínica. É necessário saber acessar a informação, avaliar a literatura, entender as limitações (vantagens e desvantagens) de cada desenho de estudo, os métodos estatísticos envolvidos com a situação clínica e como planejar pesquisas para que seus resultados sejam válidos.

Cabe ao profissional da área da saúde saber como é realizado cada um dos itens básicos, com consciência dos aspectos envolvidos no processo da tomada de decisão clínica. Dentro do contexto apresentado, percebemos que a MBE é a aplicação dos resultados das pesquisas clínicas para ori-

1. Aluno do Programa de Pós-Graduação em Cirurgia da Universidade Federal do Rio de Janeiro - RJ.

2. Supervisora do Programa de Residência Médica do Serviço de Clínica Médica do Hospital do Andaraí, Rio de Janeiro.

3. Professor Adjunto do Departamento de Cirurgia da Faculdade de Medicina da Universidade Federal do Rio de Janeiro.

4. Professor Titular de Clínica Cirúrgica do Curso de Graduação da Fundação Educacional Serra dos Órgãos, Teresópolis - RJ e Professor Adjunto do Departamento de Cirurgia da Faculdade de Medicina da Universidade Federal do Rio de Janeiro.

5. Grupo de Estudo de Revisão Sistemática do Rio de Janeiro (GERS-Rio).

Recebido em 10/09/2007

Aceito para publicação em 11/10/2007

Conflito de interesse: nenhum

Fonte de financiamento: nenhuma 
entar no processo da tomada de decisão em saúde nos mais diferentes níveis. A integração das evidências, as vivências, a competência e a ética é o que devem prevalecer.

\section{HISTÓRICO DA REVISÃO SISTEMÁTICA}

As primeiras técnicas formais da combinação dos resultados de diferentes estudos foram elaboradas e publicadas no British Medical Journal, pelo matemático britânico Karl Pearson, em 1904. Ele estudou o efeito preventivo das inoculações contra a febre entérica, ao observar que os resultados de pequenos estudos isolados não ofereciam condições para se obter conclusões sobre o assunto, sem que uma grande probabilidade de erro existisse ${ }^{2}$.

Em 1955, aparece a primeira revisão sistemática sobre um cenário clínico, publicada no Journal of American Medical Association $^{2}$. Antes dessa data, surgiram algumas publicações que abordavam os métodos estatísticos - metanálise para combinar resultados de estudos independentes. O termo metanálise surgiu, pela primeira vez, em 1976, em artigo da revista Educational Research ${ }^{3}$.

A era das revisões sistemáticas com metanálises, na área da Saúde, se consolidou no final da década de 80 com a publicação do livro Effective Care During Pregnancy and Childbirth ${ }^{4}$. Duas outras publicações da mesma época, uma em Cardiologia ${ }^{5}$ e outra em Oncologia ${ }^{6}$ tiveram grande importância.

Na década de 90, ocorre a fundação da Cochrane Collaboration <http://www.cochrane.org/index.htm>, organização internacional que surge em reconhecimento a um dos criadores da MBE, professor Archie Cochrane, pesquisador britânico e autor do livro Effectiveness and Efficiency: Random Reflections on Health Service (1972). A Cochrane Collaboration tem como objetivo preparar, manter e disseminar revisões sistemáticas na área da Saúde. Somente na Europa, instalaram-se sete centros Cochrane (França, Alemanha, Grã-Bretanha, Espanha, Itália, Holanda e Dinamarca), além de centros no Canadá, China, Austrália, Nova Zelândia, África do Sul e Brasil ${ }^{7}$.

Também na década de 90 , apareceram as duas primeiras teses que consistiam em revisões sistemáticas com metanálises: uma na Inglaterra e outra no Brasil. Em Oxford, Alejandro Jadad defendeu sua tese de Doutoramento, e, em São Paulo, Jair de Jesus Mari a de Livre Docência na Escola Paulista de Medicina, ambas em 1994. Em 1997, também na Escola Paulista de Medicina, foi defendida a primeira tese de doutoramento cujo tema foi a revisão sistemática com metanálise ${ }^{8}$.

Em 1995, um grupo de cientistas reunidos em Potsdam (Alemanha) definiu como revisão sistemática "a aplicação das estratégias científicas que limitam o viés de seleção e avaliam com espírito científico os artigos e sintetizam todos os estudos relevantes em tópicos específicos ${ }^{9}$.

Em 25 de setembro 2007, a base de dados Cochrane continha 4.777 revisões sistemáticas da Cochrane, 6.113 revisões de revisões sistemáticas com qualidade avaliada, 495.002 registros Cochrane de ensaios controlados (CENTRAL/CCTR), 11 bases de dados Cochrane de revisões de Metodologia, 9.458 registros Cochrane de Metodologia, 91 artigos sobre a Cola- boração Cochrane, 6.817 resumos do International Network of Agencies for Health Technology Assessment (INAHTA) e de outras agências de Avaliação de Tecnologia em Saúde e 21.149 avaliações econômicas do National Health Service (NHS).

O acesso à Biblioteca Cochrane na BVS < http:// www.bireme.br/php/index.php> está disponível para profissionais de saúde da América Latina e Caribe e é resultado de um acordo cooperativo entre a BIREME/OPAS, a Colaboração Cochrane e o Centro Cochrane do Brasil.

\section{REVISÃO SISTEMÁTICA}

A revisão sistemática é um tipo de investigação científica que tem por objetivo reunir, avaliar criticamente e conduzir uma síntese dos resultados de múltiplos estudos primári$\mathrm{os}^{10}$. Ela também objetiva responder a uma pergunta claramente formulada, utilizando métodos sistemáticos e explícitos para identificar, selecionar e avaliar as pesquisas relevantes, coletar e analisar dados de estudos incluídos na revisão ${ }^{11}$. Os métodos estatísticos (metanálise) podem ou não ser usados para analisar e sumarizar os resultados dos estudos incluídos.

Nas revisões sistemáticas os "sujeitos" da investigação são os estudos primários (unidades de análise) selecionados por meio de método sistemático e pré-definido. Os estudos primários podem ser ensaios clínicos aleatórios, estudos de acurácia, estudos de coortes ou qualquer outro tipo de estudo. A escolha do tipo de estudo depende da pergunta que se pretende responder. Tradicionalmente, a revisão sistemática é um estudo retrospectivo. Existe ainda a possibilidade de realizar a revisão sistemática com dados individuais ${ }^{11}$.

A escolha do tipo de estudo depende da pergunta que se pretende responder. Para a formulação da pergunta temos que inicialmente identificar o problema $(\mathrm{P})$, a intervenção (I), o que será comparado (C), qual o desfecho (D) e, eventualmente o tempo $(\mathrm{T})$ decorrido para se avaliar o desfecho.

Como em qualquer forma de pesquisa, as revisões sistemáticas são consideradas investigações científicas em si mesmas ${ }^{12} \mathrm{e}$, assim como as demais revisões, elas são qualificadas como estudos observacionais retrospectivos, por alguns autores ${ }^{10}$. Outros as situam em algum lugar entre os estudos experimentais e observacionais, não podendo ser inteiramente classificadas em nenhuma das duas categorias ${ }^{13}$.

Uma boa revisão sistemática é baseada na formulação adequada de uma pergunta. Uma pergunta bem estruturada é o começo de uma boa revisão sistemática, pois define quais serão as estratégias adotadas para identificar os estudos que serão incluídos e quais serão os dados que necessitam ser coletados de cada estudo.

\section{REVISÃO NARRATIVA}

A revisão da literatura narrativa ou tradicional, quando comparada à revisão sistemática, apresenta uma temática mais aberta; dificilmente parte de uma questão específica bem definida, não exigindo um protocolo rígido para sua confecção; a busca das fontes não é pré-determinada e específica, sendo frequentemente menos abrangente. A seleção dos artigos é arbitrária, provendo o autor de informações sujeitas a 
viés de seleção, com grande interferência da percepção subjetiva.

\section{METANÁLISE}

Entre os temas abordados neste artigo, talvez o que tenha maior problema de definição na literatura seja a metanálise. Com muita freqüência, o termo aparece significando uma revisão completa, que inclui busca na literatura, extração de dados e combinação dos dados quantitativos; outras vezes o termo é restringido à descrição da síntese quantitativa de diferentes estudos dentro de uma revisão. Há autores que descrevem metanálise como uma revisão sistemática quantitativa ${ }^{14}$; outros, mais especificamente, como a combinação estatística de pelo menos dois estudos, para produzir uma estimativa única.

Na primeira definição formal na literatura científica, metanálise é "a análise estatística de uma coleção de resultados de estudos individuais, com o objetivo de integrar os resultados" ${ }^{3}$. O termo metanálise é comumente usado para se referir às revisões sistemáticas que utilizam a metanálise.

O prefixo meta tem origem no grego e significa "além", "transcendência", "reflexão crítica sobre". A grafia da palavra freqüientemente gera discussão ${ }^{15}$. O termo meta-analysis foi incluído entre os Medical Subject Headings (PubMed) ou Descritores em Ciência da Saúde (Biblioteca Virtual em Saúde) em 1992, o que permite a utilização desse descritor para identificar metanálises publicadas na Medline e na LILACS.

Para que o produto de uma metanálise, como síntese quantitativa de uma revisão, seja fidedigno, é extremamente importante que os estudos-fonte dos dados originais tenham sido avaliados com critérios tais que se possa confiar na adequação de sua associação com a finalidade de chegar a um resultado integrado. Para isso não são suficientes apenas métodos estatísticos, pois eles não têm poder para tornar prescindível a avaliação metodológica de cada um dos estudos ${ }^{14}$.

\section{DISCUSSÃO}

Os termos revisão sistemática, metanálise e a expressão Medicina Baseada em Evidência têm sido frequen- temente utilizados na literatura médica e precisam ser melhor conceituados.

A grande quantidade de informações médicas relacionadas à terapêutica cirúrgica e o tempo limitado dos profissionais da área da Saúde trazem a necessidade do desenvolvimento de processos que proporcionem caminhos concisos até os resultados obtidos em uma pesquisa.

Diante dos grandes debates entre defensores e opositores do método, observamos um número crescente de artigos publicados onde os autores utilizam como desenho de estudo a revisão sistemática com ou sem metanálise. Na verdade, é recente a incorporação desse método pelos americanos. No entanto, autores europeus e canadenses escrevem exaustivamente sobre o assunto, principalmente no Bristish Medical Journal, no The Lancet e no Journal of the American Medical Association. No Brasil é cada dia mais presente a participação dos autores brasileiros, especialmente na área de Saúde Mental, com publicações em importantes periódicos estrangeiros.

Entendemos que seja necessária a produção de revisões sistemáticas relacionadas aos problemas cirúrgicos, o que contribuiria para as tomadas de decisão em Cirurgia.

O conceito de Medicina Baseada em Evidência deve ser discutido, para que não se cometa o equívoco de associálo à extinção da função do médico. A medicina baseada em evidência traz uma filosofia, que é a busca de um tipo de conhecimento construído a partir de informações objetivas, produzidas por intermédio de pesquisas rigorosas para a minimização dos vieses.

\section{CONCLUSÂO}

Os autores concluem com a necessidade de mais discussões sobre revisão sistemática entre os cirurgiões.

\section{AGRADECIMENTOS}

Agradecemos à Sonia M. R. Vasconcelos (UFRJ) pela revisão crítica do manuscrito.

\begin{abstract}
Purpose: Conduct a narrative review about systematic review. Data source: We searched the Cochrane Methodology Register, MEDLINE, LILACS, Google Scholar and Cochrane Library (2000 to January 2007). We also handsearched reference lists, and used personal communications. Selection of studies: This was supplemented with a search strategy in Medline using the following combinations of MeSH-terms "Meta-Analysis" [Publication Type] AND "Evidence-Based Medicine” [MeSH] Limits:Publication Date from 2000 to 2007, Humans. Systematic Reviews. In LILACS: (metanalise) or "METANALISE" [Descritor de assunto] and [medicina baseada em evidências] or "MEDICINA BASEADA EM EVIDÊNCIAS" [Descritor de assunto]. In The Cochrane Methodology Review Group and Google Scholar: "revisão sistemática e metanálise"; "medicina baseada em evidências". After independent review by two observers, ten articles that specifically addressed the stated purpose were selected. Data Synthesis: The subjects assessed in these studies were grouped into two categories, ie, those addressing the history of systematic review, and the definitions of Evidence-Based Medicine, Systematic Review, Narrative Review and Meta-Analysis. Conclusions: The authors find that further discussion on systematic review should be encouraged among surgeons.
\end{abstract}

Key words: Communication. Systematic review. Meta-analysis. Review literature. Narrative review. Evidence-Based Medicine. 


\section{REFERÊNCIAS}

1. Sackett DL, Rosenberg WMC, Gray JAM, Richardson WS. Evidence based medicine: what it is and what it isn't. BMJ. 1996;312:71-2.

2. Beecher HK. "The Powerful Placebo". JAMA. 1955 Dec;159(17):1602-6.

3. Glass GV. Primary, Secundary and Meta-Analysis of Research. Educational researcher.1976 Nov;5(10):3-8.

4. Chalmers I, Enkin M, Keirse MJNC, editores. Effective care in pregnancy and childbirth. Oxford: Oxford University Press; 1989.

5. Yusuf S, Peto R, Lewis J, Collins R, Sleight P. Beta blockade during and after myocardial infarction: an overview of the randomized trials. Progr Cardiovasc Dis. 1985;17:335-71.

6. Early Breast Cancer Trialists' Collaborative Group. Effects of adjuvant tamoxifen and of cytotoxic therapy on mortality in early breast cancer: an overview of 61 randomized trials among 28,896 women. N Engl J Med. 1988;319:1681-92.

7. Castro AA. Curso de Revisão Sistemática e Metanálise. Disponível em: http://www.virtual.epm.br/cursos/metanalise/

8. Soares KVS: Discinesia tardia induzida por neurolépticos: metanálise dos ensaios clínicos controlados [tese doutorado]. EPM: UNIFESP; 1997.

9. Perissé ARS, Gomes MM, Nogueira AS. Revisões sistemáticas (inclusive metanálises) e diretrizes clínicas. In: Gomes MM, edi- tor. Medicina baseada em evidências: princípios e práticas. Rio de Janeiro: Reichmann \& Affonso; 2001. p.131-48.

10. Cook DJ, Mulrow CD, Haynes RB. Systematic reviews: synthesis of best evidence for clinical decisions. Ann Intern Med. 1997;126(5):376-80.

11. Clarke M, Horton R. Bringing it all together: Lancet-Cochrane collaborate on systematic reviews. Lancet. 2001 Jun 2;357:1728.

12. Dixon-Woods M, Agarwal S, Jones D, Young B, Sutton A. Synthesising qualitative and quantitative evidence: a review of possible methods. J Health Serv Res Policy. 2005 Jan;10(1):4553.

13. Dawson B, Trapp RG. Bioestatística básica e clínica. $3^{\text {a }}$. ed. Rio de Janeiro: McGraw-Hill; 2003.

14. Lau J, Joannidis JPA, Schmid CH: Quantitative synthesis in systematic reviews. Ann Intern Med. 1997; 127(9): 820-6.

15. Guimarães CA. Língua Portuguesa na Medicina. São Paulo. Disponível em: http://www.metodologia.org/gramatica.pdf

Como citar este artigo:

Cordeiro AM, Oliveira GM, Renteria JM, Guimarães CA, GERSRio. Revisão sistemática : Uma revisão narrativa. Rev Col Bras Cir. [periódico na Internet] 2007; 34(6). Disponível em URL: http:// www.scielo.br/rcbc

Endereço eletrônico: GERS-Rio@yahoogroups.com 